\title{
Prioritizing the Strategies to Enhance Smart City Logistics by Intuitionistic Fuzzy CODAS
}

\author{
Gülçin BÜYÜKÖZKAN ${ }^{a}$ and Fethullah GÖÇER ${ }^{b}$ \\ ${ }^{a}$ Industrial Engineering Department, Galatasaray University, 34349 Ortakoy, Istanbul, Turkey \\ b Industrial Engineering Department, Kahramanmaras Sutcu Imam University, 46040, Kahramanmaras, Turkey
}

\begin{abstract}
Accurate, well-timed, and efficient delivery operations is a must for supply chains and logistics providers' survival in a digitally enhanced business environment. Sustainability, mobility and livability related objectives of Smart City Logistics (SCL) address the reduction of trucks, congestion and pollution. In order to accomplish this objective, all stakeholders must work together. In addition to strong collaboration among cities, supply chains, and logistics providers, digital enablers can resolve traditional challenges in last-mile logistics for sustainable, mobile and livable cities. While the approaches to enhance SCL are in abundance, prioritizing suitable SCL strategies is a non-trivial task. In order to improve the SCL and evaluate the required strategies, this paper develops a decisionmaking model based on the Combinative Distance Based Assessment (CODAS) under Intuitionistic Fuzzy (IF) setting and carries out a pioneering study to determine and prioritize the required SCL strategies. It proposes a new approach to support the claim, and analyzes the SCL for the evaluation system. Then, selection criteria are established to define SCL strategies and to develop a method based on IF CODAS and the prospect theory. Finally, the advantages, disadvantages, as well as the limitations of the proposed method are discussed. This study is one of the pioneering research with empirical contributions to the existing vastly conceptual discussion.
\end{abstract}

Keywords: Intuitionistic Fuzzy, Intuitionistic Fuzzy CODAS, DecisionMaking, Smart City Logistics, Strategy Listing.

\section{Introduction}

The majority of global population lives in cities, leading to the most complex and most influential area of logistics in cities. "Urban Logistics", which started to be examined as a sub-branch of logistics in the early 1990s, aims at planning and managing distribution and transportation as in general logistics. Smart City Logistics (SCL), on the other hand, is a relatively new concept that is gaining an increased extent of notice from scholars and practitioners. Traffic, air and noise pollution, increasing transportation costs and product price hikes have become widespread in cities with high population density and population growth rates. This is also due to the increase in the proportion of the population with privately owned vehicles and expanding transportation needs. The ongoing relocation of people to cities and escalation in online shopping will create even more deliveries to and within urban areas. If the process is left with no intervention and without any strategies to achive an optimal SCL, these increasing trends will cause even more congestion of traffic, air and noise pollution, higher carbon dioxide emissions, etc.

For this reason, it is of great importance to examine and improve SCL by accurate strategies. Improvements in SCL can have many benefits, such as less traffic, cleaner air, lower accident risk, decrease in noise and less logistics costs embedded in product prices, as well as better quality of life, effective use of resources, sustainability, economic potential and environmental protection.

In general, there are many criteria that conflict with each other in the evaluation and selection of strategies. Therefore, Multiple Criteria Decision Making (MCDM) methods can be used to solve these problems. In addition, the fuzzy set theory [19] can eliminate the uncertainty of human opinions in their subjective evaluations. The fuzzy set theory proposed by Zadeh [19] in 1965 deals with problems of uncertainty, which are the basis of fuzzy logic. This theory is used to incorporate indefinite data into the decision framework, in which the transition from membership to non-membership is gradual. This provides strong and meaningful means of measuring uncertainty, as well as a meaningful representation of 
ambiguous concepts expressed in natural language. In a fuzzy set, verbal expressions (such as low, medium, high) that qualify a certain quantity are used, depending on the degree of membership in the cluster. A significant difference from other systems of fuzzy logic is that it allows the use of verbal variables, a useful tool to capture complex and problematic concepts without traditional quantitative terms [3]. The words mentioned herein are words that cannot express the boundary condition clearly in traditional set theory. Words may involve complexity, subjectivity or uncertainty, so that a verbal variable must be defined on the basis of fuzzy sets. Thus, verbal variables ensure that the concepts that cannot be clearly expressed can be described as approximate. Atanassov [2] extended the fuzzy set theory involving non-membership and hesitation degrees in addition to the membership degree and developed the Intuitionistic Fuzzy (IF) sets theory. Its strong ability to deal with the hesitation and fuzziness makes IF logic an effective and frequently used theory in decision-making problems.

In this study, SCL strategies and the criteria to evaluate them are determined in line with the literature review and opinions of sectoral expert. The importance of the determined criteria is calculated with IF logic, and the determined strategies are prioritized by using COmbinative Distance-based Assessment (CODAS) [11] technique, developed recently as one of the latest MCDM methods. It is a distance-based technique and uses a combinative form of Euclidean and Taxicab distances. In the assesment process, the alternative having the greater distance is the most desirable candidate.

The study consists of five parts. In the second part of the study, following the introduction of the study purpose, information is given about existing methods that can be used in determining SCL strategies. The third part presents a short introduction to IF sets and their arithmetic, as well as the proposed methodology. In the fourth part of the study, an application is made to prioritize the SCL and the results are interpreted. The last part of the study includes a short evaluation.

\section{The determination of Strategies for Smart City Logistics}

Urban logistics, which is a concept put forward in the early 1990s especially in Europe and Japan, is defined by the Urban Logistics Institute as a process of optimization of the transportation activities of all the logistics and private companies in the urban areas bearing in mind the traffic environment, energy consumption and traffic congestion in free markets [16]. In summary, SCL is the best way to direct and manage the flows of goods and people entering and leaving the cities and circulating in the city. Their main objectives are global competition, efficiency, environmental protection, traffic reduction, security, safety, energy saving and effective use of labor. These objectives are related to the concepts of sustainability, mobility and livability, which are the three pillars of SCL. The aim of each improvement work carried out in SCL systems should be carried out considering these three building blocks [8].

The urban population of the world has increased rapidly from 751 million in 1950 to 4.2 billion in 2018 . The proportion of world residing in communities in provinces and districts is $55 \%$ in 2018 , this proportion is expected to incrase to $68 \%$ by 2050 [17]. Cities, however, are often unconsciously planned and inadequate to such tremendous changes of population and traffic at this scale. The traffic between the houses, businesses and other social areas in the city center and the traffic of the freight transport to these centers are intertwined, making the system more difficult to improve. Sustainability measures, such as bicycle lanes, pedestrian roads, public transport systems, and roads that have been closed to traffic, can further impede the transport of goods in cities, also due to missing parking and loading and unloading areas. In this multivariate and multi-actor system, improvement studies should be carried out carefully and in detail with all these variables and actors in mind. Strategies for enabling cities for urban logistics should be examined on the basis of different targets, i.e. evaluation criteria.

When the literature on urban logistics is examined, it is seen that the studies on this subject have intensified especially in recent years. Some of the studies are summarized as follows: Amaral and Aghezzaf [1] modeled traffic management in metropolitan areas and effective management of urban logistics strategies. In this study, the proposed optimization model and solution approaches for two-step system strategies are discussed. In another study, Erdumlu [10] examined the concepts of Urban Logistics and Logistic Village, and made a comparative evaluation of the optimum modeling methods developed within the framework of urban logistics concept. He determined the size and location of the organized logistics area in Istanbul. In a recent study, Yang et al. [18] proposed a new carbon tax-constrained urban logistics distribution network planning model using mixed-integer programming method. This study aims to determine possible SCL strategies for Istanbul with a four step methodology:

\section{Step 1. Define SCL strategies.}

Step 2. Define criteria to evaluate SCL strategies.

Step 3. Calculate the importance of the criteria to evaluate the SCL strategies defined in the second step with the IF Logic approach.

Step 4. Prioritize the SCL strategies defined in the first step based on the evaluation criteria.

The methods to be used in Step 3 and Step 4 are briefly described in the next subsections of this study. 


\section{Methodology}

\subsection{Preliminaries}

This subsection presents the basic definitions regarding to the IF set theory.

Fuzzy set theory is represented by $\mathrm{A}$ in $X$ as:

$\mathrm{A}=\left\{<x, \mu_{A}(x)>\mid x \in X\right\}$,

And IF set theory is represented by $\widetilde{\mathrm{A}}$ in $X$ as:

$\widetilde{\mathrm{A}}=\left\{<x, \mu_{\widetilde{\mathrm{A}}}(x), v_{\widetilde{\mathrm{A}}}(x)>\mid x \in X\right\}$,

Where $\mu_{\widetilde{\mathrm{A}}}(x): X \rightarrow[0,1] \quad$ and $v_{\widetilde{\mathrm{A}}}(x): X \rightarrow[0,1]$ represents the membership and non-membership degrees of $x \in X$, respectively. The IF sets should have the following condition:

$0 \leq \mu_{\widetilde{\mathrm{A}}}(x), v_{\widetilde{\mathrm{A}}}(x) \leq 1$,

$\pi_{\widetilde{\mathrm{A}}}(x)=1-\mu_{\widetilde{\mathrm{A}}}(x)-v_{\widetilde{\mathrm{A}}}(x)$.

$\pi_{\widetilde{A}}(x)$ indicates the hesitancy degree. Hence, an IF set $\widetilde{\mathrm{A}}$ in $\mathrm{X}=\{\mathrm{X}\}$ is fully defined with the form of Equation (Eq.) (2) where

$\mu_{\widetilde{\mathrm{A}}}(\mathrm{x}) \rightarrow[0,1] ; v_{\widetilde{\mathrm{A}}}(\mathrm{x}) \rightarrow[0,1] ; \pi_{\widetilde{\mathrm{A}}}(\mathrm{x}) \rightarrow[0,1]$.

Let $\widetilde{A}$ and $\widetilde{B}$ be two IF set in $X$ and $\lambda>0$. Some of the basic arithmetic operations in IF sets are defined as follows:

$\widetilde{\mathrm{A}} \oplus \widetilde{\mathrm{B}}=\left\{\left[\begin{array}{c}\mathrm{x}, \mu_{\widetilde{\mathrm{A}}}(\mathrm{x})+\mu_{\widetilde{\mathrm{B}}}(\mathrm{x}) \\ -\mu_{\widetilde{\mathrm{A}}}(\mathrm{x}) * \mu_{\widetilde{\mathrm{B}}}(\mathrm{x}), \\ \nu_{\widetilde{\mathrm{A}}}(\mathrm{x}) * \nu_{\widetilde{\mathrm{B}}}(\mathrm{x})\end{array}\right] \mid \mathrm{x} \in \mathrm{X}\right\}$,

$\widetilde{\mathrm{A}} \otimes \widetilde{\mathrm{B}}=\left\{\left[\begin{array}{c}\mathrm{x}, \mu_{\widetilde{\mathrm{A}}}(\mathrm{x}) * \mu_{\widetilde{\mathrm{B}}}(\mathrm{x}), \\ v_{\widetilde{\mathrm{A}}}(\mathrm{x})+v_{\widetilde{\mathrm{B}}}(\mathrm{x}) \\ -v_{\widetilde{\mathrm{A}}}(\mathrm{x}) * v_{\widetilde{\mathrm{B}}}(\mathrm{x})\end{array}\right] \mid \mathrm{x} \in \mathrm{X}\right\}$,

$\lambda * \widetilde{\mathrm{A}}=\left\{\left[\begin{array}{c}\mathrm{x}, 1-\left(1-\mu_{\widetilde{\mathrm{A}}}(\mathrm{x})\right)^{\lambda}, \\ v_{\widetilde{\mathrm{A}}}(\mathrm{x})^{\lambda}\end{array}\right] \mid \mathrm{x} \in \mathrm{X}\right\}$,

\subsection{IF CODAS Method}

Step 1: Determine decision criteria and alternatives

The alternatives of set $A_{i}$ with $i=1,2, \ldots, m$ each of them evaluated for decision criteria of set $C_{j}$ with $j=$ $1,2, \ldots, n$.

Step 2: Determine DMs’ weights

$k=1,2, \ldots, K$, as a set of DMs, with their individual weight $\lambda_{k}$ with $\sum_{\mathrm{k}=1}^{K} \lambda_{k}=1$. Use Eq. (8) to calculate DMs' weights, adapted from [4].

$\lambda_{k}=\frac{\left[\mu_{k}+\pi_{k}\left[\frac{\mu_{k}}{1-\pi_{k}}\right]\right]}{\sum_{k=1}^{K}\left[\mu_{k}+\pi_{k}\left[\frac{\mu_{k}}{1-\pi_{k}}\right]\right]}$, where $\sum_{k=1}^{K} \lambda_{k}=1$,
Step 3: Determine criteria weights

Let $\widetilde{w}_{j}$ define the criteria weights in the form of IF set value. The Eq. (9) is used to calculate criteria weights.

$\widetilde{w}_{j}=\mathrm{IFWA}_{\lambda}=\left(\widetilde{w}_{j}^{(1)}, \widetilde{w}_{j}^{(2)}, \ldots, \widetilde{w}_{j}^{(K)}\right)$

$\widetilde{w}_{j}=\lambda_{1} * \widetilde{w}_{j}^{(1)} \oplus \lambda_{2} * \widetilde{w}_{j}^{(2)} \oplus \ldots \oplus \lambda_{K} * \widetilde{w}_{j}^{(K)}$

$\widetilde{w}_{j}=\left(1-\prod_{k=1}^{K}\left(1-\mu_{i j}^{(k)}\right)^{\lambda_{K}}, \prod_{k=1}^{K}\left(\left(v_{i j}^{(k)}\right)^{\lambda_{K}}\right)\right)$,

Step 4. Determine individual decision matrix

The individual opinion of DM in linguistic terms are transformed by the linguistic variables in Table 2 [6] and individual decision matrix $\left(A_{(k)_{i j}}\right)_{m x n}$ is constructed.

$A_{(k)_{i j}}=\left[\begin{array}{ccc}a_{11} & \cdots & a_{1 n} \\ \vdots & \ddots & \vdots \\ a_{m 1} & \cdots & a_{m n}\end{array}\right]$,

Step 5: Determine aggregated decision matrix

The individual alternative evaluations are combined into GDM matrix by intuitionistic fuzzy weighted averaging (IFWA) aggregation operator in Eq. (11).

$$
\begin{aligned}
& \operatorname{IFWA}\left(\tilde{A}_{1}, \tilde{A}_{2}, \ldots, \tilde{A}_{k}\right)=\left(\begin{array}{c}
1-\prod_{k=1}^{K}\left(1-\mu_{i j}^{(k)}\right)^{\lambda_{K}}, \\
\prod_{k=1}^{K}\left(\left(v_{i j}^{(k)}\right)^{\lambda_{K}}\right)
\end{array}\right), \\
& A_{i j}=\left[\begin{array}{ccc}
a_{11} & \cdots & a_{1 n} \\
\vdots & \ddots & \vdots \\
a_{m 1} & \cdots & a_{m n}
\end{array}\right]
\end{aligned}
$$

Step 6. Construct the weighted-normalized decision matrix

Table 1. Linguistic Variables of IF Set

\begin{tabular}{|l|c|c|c|c|}
\hline \multicolumn{2}{|c|}{ Linguistic Terms } & $\mu$ & $v$ & $\pi$ \\
\hline Very Good & VG & 1.00 & 0.00 & 0.00 \\
\hline Good & G & 0.75 & 0.10 & 0.15 \\
\hline $\begin{array}{l}\text { Medium } \\
\text { Good }\end{array}$ & MG & 0.60 & 0.30 & 0.10 \\
\hline Medium & M & 0.50 & 0.45 & 0.05 \\
\hline $\begin{array}{l}\text { Medium } \\
\text { Poor }\end{array}$ & MP & 0.30 & 0.50 & 0.20 \\
\hline Poor & P & 0.15 & 0.70 & 0.15 \\
\hline Very Poor & VP & 0.00 & 0.90 & 0.10 \\
\hline
\end{tabular}


Normalize the decision matrix by swapping the values of membership and non-membership in the case of cost-type criteria and use Eq. (13) to find the weighted matrix. Weights are given in Step 3.

$\tilde{R}_{i j}=\left\{\tilde{r}_{i j}\right\}=\widetilde{w}_{j} \otimes \tilde{x}_{i j}$,

where $\quad \tilde{r}_{i j}=\left(\mu_{\mathrm{ij}}, v_{\mathrm{ij}}, \pi_{\mathrm{ij}}\right), \quad j=1,2,3, \ldots, n, \quad i=$ $1,2,3, \ldots, m$,

Step 7. Determine the negative ideal solutions.

Use Eq. (14) to find negative $\left(A^{-}\right)$ideal solutions, respectively.

$A^{-}=\left(\tilde{r}_{1}^{-}, \tilde{r}_{2}^{-}, \ldots, \tilde{r}_{n}^{-}\right), \tilde{r}_{j}^{-}=\left(\mu_{j}^{-}, v_{j}^{-}, \pi_{j}^{-}\right)$,

Where, $j=1,2, \ldots, n$,

$\mu_{j}^{-}=\left\{\left(\min _{i}\left\{\mu_{i j}\right\} \mid j \in J_{1}\right)\right\},\left\{\left(\max _{i}\left\{\mu_{i j}\right\} \mid j \in J_{2}\right)\right\}$,

$v_{j}^{-}=\left\{\left(\max _{i}\left\{v_{i j}\right\} \mid j \in J_{1}\right)\right\},\left\{\left(\min _{i}\left\{v_{i j}\right\} \mid j \in J_{2}\right)\right\}$,

$\pi_{j}^{-}=1-\mu_{j}^{-}-v_{j}^{-}$,

Suppose $J_{1}$ is the benefit criteria, $J_{2}$ is the cost criteria.

Step 8. Determine the Euclidean distance and the Hamming distance

The Euclidean distance:

$E\left(\tilde{R}_{i j}, A^{-}\right)=\sqrt{\frac{1}{2} \sum_{j=1}^{n}\left[\begin{array}{c}\left(\mu_{\mathrm{ij}}-\mu_{j}^{-}\right)^{2}+ \\ \left(v_{\mathrm{ij}}-v_{j}^{-}\right)^{2}+ \\ \left(\pi_{\mathrm{ij}}-\pi_{j}^{-}\right)^{2}\end{array}\right]}$

The Hamming distance:

$H\left(\tilde{R}_{i j}, A^{-}\right)=\frac{1}{2} \sum_{j=1}^{n}\left(\begin{array}{c}\left|\mu_{\mathrm{ij}}-\mu_{j}^{-}\right|+ \\ \left|v_{\mathrm{ij}}-v_{j}^{-}\right|+ \\ \left|\pi_{\mathrm{ij}}-\pi_{j}^{-}\right|\end{array}\right)$

Step 9. Establish relative assessment matrix

Relative assessment matrix $\left(R A_{i j}=\left\{r a_{i j}\right\}_{m x m}\right)$ is constructed by the formula in Eq. (17).

$r a_{i l}=\left(\begin{array}{c}{\left[E\left(\tilde{R}_{i j}, A^{-}\right)-E\left(\tilde{R}_{l j}, A^{-}\right)\right]+} \\ \left(\begin{array}{c}\varphi\left[E\left(\tilde{R}_{i j}, A^{-}\right)-E\left(\tilde{R}_{l j}, A^{-}\right)\right] * \\ {\left[H\left(\tilde{R}_{i j}, A^{-}\right)-H\left(\tilde{R}_{l j}, A^{-}\right)\right]}\end{array}\right)\end{array}\right)$

where, $l=1,2, \ldots, m$, and

$\varphi\left[E\left(\tilde{R}_{i j}, A^{-}\right)-E\left(\tilde{R}_{l j}, A^{-}\right)\right]=$

$\left\{\begin{array}{llc}1 & \text { if } & |\tau| \leq E\left(\tilde{R}_{i j}, A^{-}\right)-E\left(\tilde{R}_{l j}, A^{-}\right) \\ 0 & \text { if } & \text { others }\end{array}\right.$

and $\varphi$ represents the threshold function of the equality of the Euclidean distance, $\tau$ is the threshold value set by the DMs, and $r a_{i l}$ represent the priority difference of each alternative [13].

Step 10. Rank Alternatives

The final score of each alternatives is estimated with Eq. (19).

$\delta_{i}=\sum_{l=1}^{m} r a_{i l}$

The alternatives are ranked in a descending order of the final score $\left(\delta_{i}\right)$.

\section{Application}

In this section, four steps of the proposed method are implemented.

\subsection{Defining SCL Strategies}

In line with the detailed literature review and expert opinions, five possible SCL strategies are identified based on their applicability in the short term:

Strategy 1: Increasing the overnight delivery rate (thus reducing the density of freight transport within the city during the day) $[5,12]$.

Strategy 2: Increasing the use of intelligent transport systems (enabling traffic management) [14].

Strategy 3: Consolidation of intra-city flows (Conducting consolidated delivery with fewer vehicles) [9].

Strategy 4: Increasing the use of electric vehicles and rail systems (By reducing the proportion of road transport, the aim is more sustainable environmentally friendly transport system) $[5,15]$.

Strategy 5: Development and use of transport network system based on intermodal transportation system (leading to the establishment of a more efficient transportation system through the transfer of rail systems from urban logistics terminals to logistics villages) [5].

\subsection{Defining evaluation criteria}

For the evaluation of SCL strategies, 6 evaluation criteria have been defined in accordance with a detailed literature review $[5,9,12,14,15]$ and expert opinions:

$C_{1}$ : Impact on environmental sensitivity (increase)

$\mathrm{C}_{2}$ : Effect on logistics costs (decrease)

$C_{3}$ : Effect on time delivery (increase)

$C_{4}$ : The effect of logistic service quality (increase)

$C_{5}$ : Effect on speed and traffic density in transport modes (decrease)

$C_{6}$ : Impact on sustainability (increase) 


\subsection{The prioritization of SCL strategies}

The SCL strategy evaluation process is applied by carrying out the following steps:

Step 1: Five commeasurable candidates are evaluated using six decision criteria to prioritize the suitable SCL strategies.

Step 2: The individual priority weights of each DM is calculated with Eq. (8). The results are displayed in Table 2. The highest importance is given to the first DM and least importance to the third DM.

Step 3: The weight of each criterion is estimated with Eq. (9) and the resulting outcome is displayed in Table 3 . The highest importance is given to the fourth criterion $\left(\boldsymbol{C}_{\mathbf{4}}\right)$ and least importance is given to the sixth criterion $\left(\boldsymbol{C}_{6}\right)$.

Step 4: DMs' judgments are presented in Table 4. Using linguistic terms in Table 1, DMs' ratings are transformed into IF values. The individual decision $\operatorname{matrix}\left(X_{(k)_{i j}}\right)_{m x n}$ with $\mathrm{m}$ alternatives and $\mathrm{n}$ criteria is thus constructed.

Step 5: The IF values are combined into GDM matrix with the IFWA operator in Eq. (11). The aggregated GDM matrix is displayed in Table 5.

Step 6: Weighted decision matrix is estimated by the criteria weights calculated in Step 3 and scalar multiplication operation of IF values based on the Eq. (7). The outcome is presented in Table 6.

Step 7: The negative ideal solutions of benefit-type and cost-type criteria for each alternative are calculated with Eq. (14). The result is provided in Table 7.

Table 2. The Weights of Each DM

\begin{tabular}{|c|c|c|c|}
\hline DM & $\mathbf{1}$ & $\mathbf{2}$ & $\mathbf{3}$ \\
\hline Term & VG & G & MG \\
\hline$\lambda_{\boldsymbol{k}}$ & 0.392 & 0.346 & 0.262 \\
\hline
\end{tabular}

Table 3. The Criteria Weights

\begin{tabular}{|c|c|c|c|}
\hline Criteria & $\mu_{\widetilde{\mathrm{A}}}(\mathrm{x})$ & ${ }_{\widetilde{\mathrm{A}}}(\mathrm{x})$ & Rank \\
\hline $\boldsymbol{C}_{\mathbf{1}}$ & 0.230 & 0.709 & 5 \\
\hline $\boldsymbol{C}_{\mathbf{2}}$ & 0.425 & 0.418 & 2 \\
\hline $\boldsymbol{C}_{\mathbf{3}}$ & 0.310 & 0.546 & 4 \\
\hline $\boldsymbol{C}_{\mathbf{4}}$ & 0.464 & 0.355 & 1 \\
\hline $\boldsymbol{C}_{\mathbf{5}}$ & 0.383 & 0.493 & 3 \\
\hline $\boldsymbol{C}_{\mathbf{6}}$ & 0.204 & 0.732 & 6 \\
\hline
\end{tabular}

Table 4. Ratings of Alternatives by DMs

\begin{tabular}{|c|c|c|c|c|c|c|c|}
\hline DM & & $C_{1}$ & $C_{2}$ & $C_{3}$ & $C_{4}$ & $C_{5}$ & $C_{6}$ \\
\hline \multirow{5}{*}{1} & $S_{1}$ & MP & VP & $\mathrm{M}$ & VP & $\mathrm{VP}$ & $\bar{G}$ \\
\hline & $S_{2}$ & $\bar{M}$ & VP & $\mathrm{P}$ & VP & $\mathrm{M}$ & $\mathrm{M}$ \\
\hline & $S_{3}$ & G & $\mathrm{MG}$ & $\mathrm{MG}$ & MP & M & MP \\
\hline & $S_{4}$ & $\mathrm{G}$ & $\mathrm{M}$ & $\mathrm{P}$ & VP & MP & $\mathrm{P}$ \\
\hline & $S_{5}$ & VP & $\mathrm{M}$ & MP & VG & $\sqrt{\mathrm{V}}$ & VP \\
\hline \multicolumn{2}{|c|}{ Criteria } & $\mathrm{M}$ & $\mathrm{G}$ & MP & $\mathrm{G}$ & MG & $\mathrm{P}$ \\
\hline \multirow{5}{*}{2} & $S_{1}$ & M & $\mathrm{P}$ & MP & $\bar{G}$ & MP & $\mathrm{P}$ \\
\hline & $S_{2}$ & 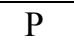 & $\mathrm{MG}$ & VP & VG & $\mathrm{P}$ & $\mathrm{VG}$ \\
\hline & $S_{3}$ & VP & VP & $\bar{P}$ & VP & $\mathrm{P}$ & $\mathrm{G}$ \\
\hline & $S_{4}$ & $r$ & G & $\mathrm{M}$ & $\mathrm{P}$ & $\mathrm{P}$ & $\mathrm{M}$ \\
\hline & $S_{5}$ & MP & $\mathrm{P}$ & $P$ & $\mathrm{VG}$ & VG & $\mathrm{P}$ \\
\hline \multicolumn{2}{|c|}{ Criteria } & MP & $\mathrm{MG}$ & $\mathrm{G}$ & $\bar{G}$ & $\mathrm{G}$ & $\mathrm{M}$ \\
\hline \multirow{5}{*}{3} & $S_{1}$ & $\mathrm{MG}$ & VG & $\mathrm{P}$ & $\mathrm{P}$ & MP & VP \\
\hline & $S_{2}$ & MP & $\mathrm{G}$ & $\mathrm{M}$ & $\mathrm{MG}$ & VP & $\mathrm{P}$ \\
\hline & $S_{3}$ & $P$ & MP & VP & $\mathrm{M}$ & G & $\mathrm{P}$ \\
\hline & $S_{4}$ & VP & $\mathrm{P}$ & $\mathrm{P}$ & $\mathrm{P}$ & MG & $\mathrm{P}$ \\
\hline & $S_{5}$ & MP & $\mathrm{G}$ & $\mathrm{MG}$ & VP & $\mathrm{VP}$ & $\mathrm{P}$ \\
\hline \multicolumn{2}{|c|}{ Criteria } & N & $\mathrm{G}$ & MG & $\mathrm{G}$ & MG & $\overline{\mathrm{MG}}$ \\
\hline
\end{tabular}

Table 5. Aggregated Decision Matrix

\begin{tabular}{|l|l|c|c|c|c|c|}
\hline & & $\boldsymbol{S}_{\mathbf{1}}$ & $\boldsymbol{S}_{\mathbf{2}}$ & $\boldsymbol{S}_{\mathbf{3}}$ & $\boldsymbol{S}_{\mathbf{4}}$ & $\boldsymbol{S}_{\mathbf{5}}$ \\
\hline $\boldsymbol{C}_{\mathbf{1}}$ & $\mu_{\widetilde{\mathrm{A}}}(\mathrm{x})$ & 0.234 & 0.180 & 0.254 & 0.260 & 0.085 \\
\hline & $\nu_{\widetilde{\mathrm{A}}}(\mathrm{x})$ & 0.685 & 0.754 & 0.599 & 0.592 & 0.823 \\
\hline $\boldsymbol{C}_{\mathbf{2}}$ & $\mu_{\widetilde{\mathrm{A}}}(\mathrm{x})$ & 1.000 & 0.241 & 0.197 & 0.304 & 0.260 \\
\hline & $\nu_{\widetilde{\mathrm{A}}}(\mathrm{x})$ & 0.000 & 0.649 & 0.722 & 0.582 & 0.642 \\
\hline $\boldsymbol{C}_{\mathbf{3}}$ & $\mu_{\widetilde{\mathrm{A}}}(\mathrm{x})$ & 0.188 & 0.097 & 0.187 & 0.142 & 0.171 \\
\hline & $\nu_{\widetilde{\mathrm{A}}}(\mathrm{x})$ & 0.741 & 0.846 & 0.737 & 0.797 & 0.732 \\
\hline $\boldsymbol{C}_{\mathbf{4}}$ & $\boldsymbol{\mu}_{\widetilde{\mathrm{A}}}(\mathrm{x})$ & 0.201 & 1.000 & 0.131 & 0.040 & 1.000 \\
\hline & $\nu_{\widetilde{\mathrm{A}}}(\mathrm{x})$ & 0.669 & 0.000 & 0.791 & 0.896 & 0.000 \\
\hline $\boldsymbol{C}_{\mathbf{5}}$ & $\boldsymbol{\mu}_{\widetilde{\mathrm{A}}}(\mathrm{x})$ & 0.085 & 0.150 & 0.260 & 0.171 & 1.000 \\
\hline & $v_{\widetilde{\mathrm{A}}}(\mathrm{x})$ & 0.823 & 0.800 & 0.642 & 0.732 & 0.000 \\
\hline $\boldsymbol{C}_{\mathbf{6}}$ & $\boldsymbol{\mu}_{\widetilde{\mathrm{A}}}(\mathrm{x})$ & 0.260 & 1.000 & 0.256 & 0.142 & 0.040 \\
\hline & $\nu_{\widetilde{\mathrm{A}}}(\mathrm{x})$ & 0.592 & 0.000 & 0.595 & 0.797 & 0.896 \\
\hline
\end{tabular}

Step 8: The Euclidean distance and Hamming distance are calculated with Eq. (15) and Eq. (16), respectively. The results are displayed in Table 8 . 
Table 6. Weighted Decision Matrix

\begin{tabular}{|l|c|c|c|c|c|c|}
\hline & & $\boldsymbol{S}_{\mathbf{1}}$ & $\boldsymbol{S}_{\mathbf{2}}$ & $\boldsymbol{S}_{\mathbf{3}}$ & $\boldsymbol{S}_{\mathbf{4}}$ & $\boldsymbol{S}_{\mathbf{5}}$ \\
\hline $\boldsymbol{C}_{\mathbf{1}}$ & $\mu_{\widetilde{\mathrm{A}}}(\mathrm{x})$ & 0.054 & 0.041 & 0.059 & 0.060 & 0.020 \\
\hline & $\nu_{\widetilde{\mathrm{A}}}(\mathrm{x})$ & 0.908 & 0.928 & 0.884 & 0.881 & 0.949 \\
\hline $\boldsymbol{C}_{\mathbf{2}}$ & $\mu_{\widetilde{\mathrm{A}}}(\mathrm{x})$ & 0.000 & 0.276 & 0.307 & 0.247 & 0.273 \\
\hline & $\nu_{\widetilde{\mathrm{A}}}(\mathrm{x})$ & 1.000 & 0.559 & 0.533 & 0.595 & 0.570 \\
\hline $\boldsymbol{C}_{\mathbf{3}}$ & $\mu_{\widetilde{\mathrm{A}}}(\mathrm{x})$ & 0.058 & 0.030 & 0.058 & 0.044 & 0.053 \\
\hline & $\nu_{\widetilde{\mathrm{A}}}(\mathrm{x})$ & 0.883 & 0.930 & 0.881 & 0.908 & 0.878 \\
\hline $\boldsymbol{C}_{\mathbf{4}}$ & $\mu_{\widetilde{\mathrm{A}}}(\mathrm{x})$ & 0.310 & 0.000 & 0.367 & 0.416 & 0.000 \\
\hline & $\nu_{\widetilde{\mathrm{A}}}(\mathrm{x})$ & 0.484 & 1.000 & 0.439 & 0.381 & 1.000 \\
\hline $\boldsymbol{C}_{\mathbf{5}}$ & $\mu_{\widetilde{\mathrm{A}}}(\mathrm{x})$ & 0.033 & 0.058 & 0.100 & 0.065 & 0.383 \\
\hline & $\nu_{\widetilde{\mathrm{A}}}(\mathrm{x})$ & 0.911 & 0.898 & 0.819 & 0.864 & 0.493 \\
\hline $\boldsymbol{C}_{\mathbf{6}}$ & $\mu_{\widetilde{\mathrm{A}}}(\mathrm{x})$ & 0.053 & 0.204 & 0.052 & 0.029 & 0.008 \\
\hline & $\nu_{\widetilde{\mathrm{A}}}(\mathrm{x})$ & 0.891 & 0.732 & 0.892 & 0.946 & 0.972 \\
\hline
\end{tabular}

Table 7. Positive and Negative Ideal Solutions

\begin{tabular}{|c|c|c|}
\hline$A^{-}$ & $\mu_{\widetilde{\mathrm{A}}}(\mathrm{x})$ & $\nu_{\widetilde{\mathrm{A}}}(\mathrm{x})$ \\
\hline $\boldsymbol{C}_{\mathbf{1}}$ & 0.020 & 0.949 \\
\hline $\boldsymbol{C}_{\mathbf{2}}$ & 0.307 & 0.533 \\
\hline $\boldsymbol{C}_{\mathbf{3}}$ & 0.030 & 0.930 \\
\hline $\boldsymbol{C}_{\mathbf{4}}$ & 0.416 & 0.381 \\
\hline $\boldsymbol{C}_{\mathbf{5}}$ & 0.033 & 0.911 \\
\hline $\boldsymbol{C}_{\mathbf{6}}$ & 0.008 & 0.972 \\
\hline
\end{tabular}

Table 8. The Euclidean distance and Hamming distance

\begin{tabular}{|c|c|c|c|c|c|}
\hline & $\boldsymbol{S}_{\mathbf{1}}$ & $\boldsymbol{S}_{\mathbf{2}}$ & $\boldsymbol{S}_{\mathbf{3}}$ & $\boldsymbol{S}_{\mathbf{4}}$ & $\boldsymbol{S}_{\mathbf{5}}$ \\
\hline$E\left(\tilde{R}_{i j}, A^{-}\right)$ & 0.434 & 0.591 & 0.140 & 0.099 & 0.673 \\
\hline$H\left(\widetilde{R}_{i j}, A^{-}\right)$ & 0.742 & 0.937 & 0.346 & 0.225 & 1.126 \\
\hline
\end{tabular}

Table 9. The relative assessment matrix

\begin{tabular}{|c|c|c|c|c|c|}
\hline & $\boldsymbol{S}_{\mathbf{1}}$ & $\boldsymbol{S}_{\mathbf{2}}$ & $\boldsymbol{S}_{\mathbf{3}}$ & $\boldsymbol{S}_{\mathbf{4}}$ & $\boldsymbol{S}_{\mathbf{5}}$ \\
\hline $\boldsymbol{S}_{\mathbf{1}}$ & 0.000 & -0.353 & 0.689 & 0.851 & -0.623 \\
\hline $\boldsymbol{S}_{\mathbf{2}}$ & 0.353 & 0.000 & 1.042 & 1.204 & -0.271 \\
\hline $\boldsymbol{S}_{\mathbf{3}}$ & -0.689 & -1.042 & 0.000 & 0.041 & -1.312 \\
\hline $\boldsymbol{S}_{\mathbf{4}}$ & -0.851 & -1.204 & -0.041 & 0.000 & -1.475 \\
\hline $\boldsymbol{S}_{\mathbf{5}}$ & 0.623 & 0.271 & 1.312 & 1.475 & 0.000 \\
\hline
\end{tabular}

Table 10. The assessment score and ranking of alternatives

\begin{tabular}{|c|c|c|}
\hline Strategy & $\boldsymbol{\delta}_{\boldsymbol{i}}$ & Ranking \\
\hline $\boldsymbol{S}_{\mathbf{1}}$ & 0.564 & 3 \\
\hline $\boldsymbol{S}_{\mathbf{2}}$ & 2.328 & 2 \\
\hline $\boldsymbol{S}_{\mathbf{3}}$ & -3.002 & 4 \\
\hline $\boldsymbol{S}_{\mathbf{4}}$ & -3.571 & 5 \\
\hline $\boldsymbol{S}_{\mathbf{5}}$ & 3.681 & 1 \\
\hline
\end{tabular}

Step 9: The relative assessment matrix is calculated with Eq. (17). The results are displayed in Table 9. It should be noted that calculations are performed by setting the threshold value to $0.05(\tau=0.05)$.

Step 10: Commeasurable candidates are ranked by following the procedure in Step 10 of the methodology. Table 10 displays the rankings that indicate the fifth alternative as the best one and the fourth alternative as the last one in the ranking.

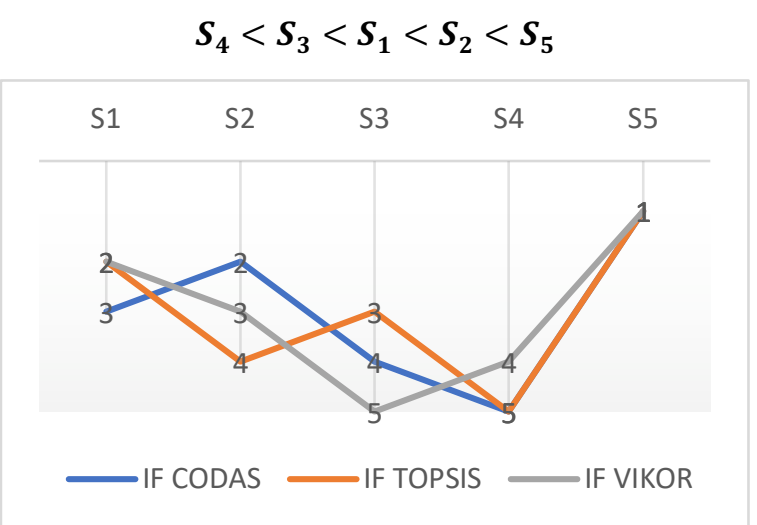

Figure 1. The analysis of the result under different MCDM techniques.

In order to assess the validity and the stability of the proposed method, a comparative analysis is also performed. For implementing the comparison, two commonly applied distance based MCDM techniques are used to analyze the results of the given outcome with the result of the proposed method. The chosen MCDM techniques are IF TOPSIS and IF VIKOR. It should be noted that these techniques has been developed in several versions, and the version considered in the analyses of Büyüközkan and Göçer [6] for TOPSIS and Büyüközkan et al. [7] for VIKOR. The results are presented in Figure 1. Therefore, the validity and robustness of the outcome of the IF CODAS approach can be confirmed.

\section{Conclusion}

The aim of this study is to determine and prioritize SCL strategies in order to decide where to start with 
improvement works in order to make cities more sustainable, more easier to move people and goods and more livable. A four-step methodology is proposed; prioritized SCL strategies are determined by evaluating the strategies and criteria that are identified with a literature review and DMs' opinions. According to the results, the criteria of the "Effect on speed and traffic density in transport modes", "Effect on logistics costs", and "Impact on environmental sensitivity" are the ones standing out as the higher importance among the six evaluation criteria. "Development and use of transport network system based on intermodal transportation system", "Increasing the use of electric vehicles and rail systems", and "Increasing the use of intelligent transport systems" are those SCL strategies that should be primarily implemented.

As a follow-up research, the number of alternative SCL strategies and evaluation criteria defined in the this study can be increased and the evaluations can be repeated with more expert opinions.

\section{Acknowledgements}

The authors would like to thank the Galatasaray University Research Fund and Kahramanmaraş Sütçü İmam University for their financial support. The authors would like to thank the experts who provide support at every stage of the study for their valuable contributions.

\section{References}

[1] R.R. Amaral, E.-H. Aghezzaf, City Logistics and Traffic Management: Modelling the Inner and Outer Urban Transport Flows in a Twotiered System, Transp. Res. Procedia. 6 (2015) 297-312.

[2] K.. Atanassov, Intuitionistic fuzzy sets, Fuzzy Sets Syst. 20 (1986) 87-96.

[3] R.E. Bellman, L.A. Zadeh, Decision-Making in a Fuzzy Environment, Manage. Sci. 17 (1970) B-141-B-164.

[4] F.E. Boran, S. Genç, M. Kurt, D. Akay, A multi-criteria intuitionistic fuzzy group decision making for supplier selection with TOPSIS method, Expert Syst. Appl. 36 (2009) 11363-11368.

[5] G. Büyüközkan, O. Feyzioğlu, F. Göçer, Selection of sustainable urban transportation alternatives using an integrated intuitionistic fuzzy Choquet integral approach, Transp. Res. Part D Transp. Environ. 58 (2018) 186-207.

[6] G. Büyüközkan, F. Gocer, An Intuitionistic Fuzzy MCDM Approach for Effective Hazardous Waste Management, in: Intell. Syst. Environ. Manag. Theory Appl., Springer, 2017: pp. 21-40.
[7] G. Büyüközkan, F. Göçer, Y. Karabulut, A new group decision making approach with IF AHP and IF VIKOR for selecting hazardous waste carriers, Measurement. 134 (2019) 6682.

[8] G. Büyüközkan, M. Sonçül, M. Tanyaş, Kentsel Lojistik Yapı Tasarımının Etkinleştirilmesi, Lojistik Derg. 24 (2012) 24 33.

[9] H. Eitzen, F. Lopez-Pires, B. Baran, F. Sandoya, J.L. Chicaiza, A multi-objective two-echelon vehicle routing problem. An urban goods movement approach for smart city logistics, in: 2017 XLIII Lat. Am. Comput. Conf., IEEE, 2017: pp. 1-10.

[10] R.M. Erdumlu, Kentsel Lojistik Ve Lojistik Köy Uygulaması, Istanbul Technical University, 2006.

[11] M.K. Ghorabaee, E.K. Zavadskas, Z.Z. Turskis, J. Antucheviciene, A new combinative distance-based assessment (CODAS) method for multi-criteria decisionmaking, Econ. Comput. Econ. Cybern. Stud. Res. 50 (2016) 25-44.

[12] J.M. Gutierrez, M. Jensen, T. Riaz, Applied Graph Theory to Real Smart City Logistic Problems, Procedia Comput. Sci. 95 (2016) 40-47.

[13] J. Ren, Sustainability prioritization of energy storage technologies for promoting the development of renewable energy: A novel intuitionistic fuzzy combinative distancebased assessment approach, Renew. Energy. 121 (2018) 666-676.

[14] Rose, Bell, Autry, Cherry, Urban Logistics: Establishing Key Concepts and Building a Conceptual Framework for Future Research, Transp. J. 56 (2017) 357.

[15] V. Schau, S. Apel, K. Gebhard, M. Mauch, W. Rossak, ICT-Systems for Electric Vehicles Within Simulated and Community Based Environments, in: Commun. Comput. Inf. Sci., 2016: pp. 217-222.

[16] E. Taniguchi, R.G. Thompson, City Logistics 1: New Opportunities and Challenges (Systems and Industrial Engineering), Inst. City Logist. (2018) 1-405.

[17] D./ P.D. United Nations, The World Population Projected to Live in Urban Areas, United Nations Depeartment Econ. Soc. Aff. (2018).

[18] J. Yang, J. Guo, S. Ma, Low-carbon city logistics distribution network design with resource deployment, J. Clean. Prod. 119 (2016) 223-228.

[19] L.A. Zadeh, Fuzzy sets, Inf. Control. 8 (1965) 338-353. 